\title{
Horror Infiniti.
}

Die Zigeuner als Europas Trickster

\section{Horror Infiniti. \\ The Gypsies as Europes Tricksters}

\section{Leonardo Piasere}

\begin{abstract}
:
The figure of trickster is well known to all folklore traditions round the world, even among Romani speaking groups as beng (devil) like in the Mediterranean and Balkan context. But the focus of the article concerns the transformation of Roma and other Gypsies themselves into tricksters, which can be shown in three examples: 1) Gypsies in the literature of the Italian Renaissance, 2) Gypsies in the Rumanian Ţsiganiada of the early 19th century and 3) the Gypsy cliché in the ethnography of the 20th century, which comes near to the bricoleur of Lévi-Strauss or to an indefinable entity comparable with the irrational numbers in mathematics.
\end{abstract}

Keywords: Trickster; folklore; chaos; creativity Trickster; Folklore; Chaos; Kreativität

Leonardo Piasere is professor for Cultural Anthropology at Verona University, did fieldwork among Xoraxane in North Italy and among Kalderash in Rumania and wrote numerous books and articels on Gypsies in Italy and other parts of Europe.

E-Mail: leonardo.piasere@univr.it

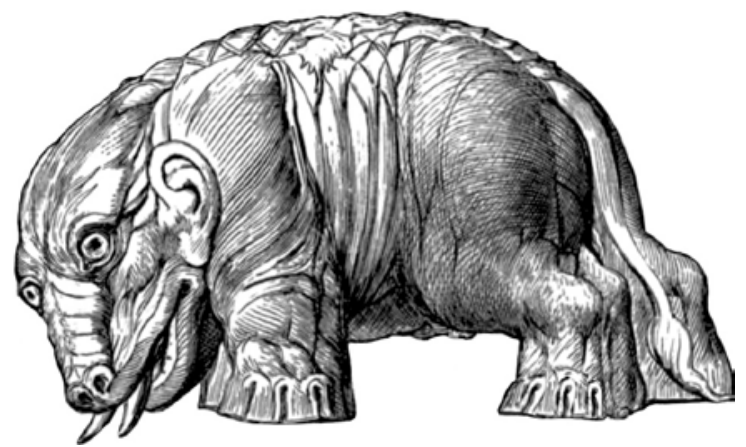


Die Figur des Trickster hat seit Daniel Brinton (1868) recht häufig die Aufmerksamkeit der Forscher auf sich gezogen. Viele Ethnologen, Volkskundler und Religionswissenschaftler haben sich mit dem Thema beschäftigt: von Boas (1898) bis Lévi-Strauss (1958), von Lowie (1909) bis Evans-Pritchard (1967), von Herskovits (Herskovits/Herskovits 1958) bis Griaule (Griaule/ Dieterlen 1965), von Pettazoni (1953) bis Eliade (1969), von Roheim (1950) bis Meletinski (1973) etc. Ohnejeden Zweifel ist der Trickster aber mit dem 1954 in Zürich veröffentlichten und bald in mehrere Sprachen übersetzten Werk „Der göttliche Schelm“ zu einer weltweit bekannten Figur geworden. In diesem Werk berichtet Paul Radin von den Abenteuern Wakdjũnkagas, des mythischen Helden der Winnebago, und beschreibt seine Charaktereigenschaften, wozu Karl Kerényi und Gustav J ung berühmte Kommentare beigesteuert haben. Die amerikanische Version von 1956 trägt den Titel „The Trickster", und dieser Begriff hat sich seitdem in der internationalen Ethnologie/ Anthropologie durchgesetzt.

Bekanntlich kommt die Figur des Trickster in Erzählungen aller Kontinente vor. Wie ich in einer kürzlich erschienen Studie zu zeigen versuchte, erscheint diese Figur auch in der Mythologie diverser Roma-Gruppen, und zwar in der Person des beng (Teufel), eines Trickster-Teufels wie man ihn ähnlich auch in anderen Traditionen des Mittelmeers und des Balkans findet (Piasere 2009). Im Rahmen dieses Artikels möchte ich nicht vom Trickster der Roma sprechen, sondern stattdessen zeigen, dass die Roma und Sinti in verschiedenen europäischen Traditionen selber zu Trickstern gemacht wurden, indem man sie als „Zigeuner“ bezeichnete. Dabei möchte ich drei Punkte untersuchen: 1) Die Zigeuner oder Quasi-Zigeuner in den komödiantischen Heldengedichten des italienischen Rinascimento sowie die Zigeuner-Frauen in den volkstümlichen Werken im Italien des 16. bis 18. Jahrhunderts, genannt Zingaresche; 2) Die Zigeuner, die in der Ţiganiada auftauchen, einem Gedicht des transsilvanischen Autors Ion Budai-Deleanu zu Beginn des 19. J ahrhunderts; 3) Die Zigeuner der transnationalen Ethnologie des 20. Jahrhunderts. Aber bevor ich auf diese Punkte eingehe, möchte ich einen kurzen Überblick über einige Interpretationen liefern, welche die „Tricksterologie" hervorgebracht hat.

Wer oder was ist der Trickster? Diese Frage ist schwer zu beantworten, denn dieses Wesen [entité] lebt von Paradoxen, wobei das erste Paradox bereits ist, ein Wesen „gegen“ die Identität zu sein. Selbst wenn er anfangs als Tier dargestellt wurde, ist er „immer mehr oder etwas anderes als das, womit man ihn gleichsetzt" (Miceli 2000, 74). Daraus folgt die gedankliche Unvorstellbarkeit dieser Person: Sie ist oft männlichen Geschlechts, kann sich aber in eine Frau verwandeln, gebären und 
wieder ein Mann werden. Ihre Verwandlungsfähigkeit ist mächtig und sie kann ständig Aussehen und Wesen verändern, wie im Fall des beng der Roma kalderaša. Siekann sich - und das interessiert uns besonders - aus Teilen mehrerer Lebewesen zusammensetzen und ein transspezifisches Lebewesen werden. Zusammengefasst hat „ihr Körper keine klaren Grenzen“ (Miceli 2000, 76). Bereits Kerényi unterstrich, dass „ihre Natur, Feind jeder Begrenzung, nach allen Richtungen offen ist“ (2006, 151). Diese physische Unvorstellbarkeit wird von einer psychologischen begleitet, denn der Trickster ist gewieft und gleichzeitig dumm, er spielt die ganze Zeit Streiche (daher sein englischer Name) und ihm werden ständig Streiche gespielt, er ist exzessiv in allem, was er tut, vom Sex bis zur Nahrung. Er kann auch ein Demiurg sein, was ihm die Macht gibt, die Welt zu schaffen bzw. zu ihrer Schaffung beizutragen - oder sie zu zerstören. Er macht alles und jeden lächerlich und ist gleichzeitig selbst lächerlich. Insgesamt ist der Trickster „Gott, Tier, Mensch, Held, Narr, derjenige, der das Gute und das Böse gelebt hat, Verneinung, Bejahung, Zerstörer und Erschaffer“ (Radin 2006, 133).

Ich werde jetzt einen Weg zur Interpretation einschlagen, der von dem kanadischen Ethnologen Rémy Savard eröffnet und von der italienischen Ethnologin Silvana Miceli weiterentwickelt wurde. Savard behauptete, der Trickster der nordamerikanischen Algonquin, den sie Glouton nannten, stehe für Schwingungen „zwischen Kontinuität und Diskontinuität“ (Savard 1978, 30) und sei der wellenförmige Erschaffer „von Vielfalt, ausgehend von einem Kontinuum“ (ebd., 36). Diese Qualität des Trickster führt laut Silvana Miceli zu seiner „speziellen Fähigkeit, in sich alle Arten von Widersprüchen und entgegengesetzten Polen (auf allen möglichen Ebenen) aufzunehmen“ (Miceli 2000, 72). Daher rühren die ihn auszeichnenden Eigenschaften wie „Widerspruch und Unordnung“ (ebd., 77), seine Unanständigkeit als „Verletzer der Normen“ (ebd., 85) - alles in allem seine „kohärente Widersprüchlichkeit“. Die „Wesensgleichheit des Trickster mit der Unordnung“ (ebd., 77) macht ihn zum „einsamen Erkunder der Unordnung“ (ebd., 113) par excellence. Diese sehr schöne Definition möchte ich als Ausgangspunkt nehmen, um meine eigenen Überlegungen darzulegen. Da die Silbe „Un-“ in „Unordnung“ pejorativ ist, bevorzuge ich es, von Nicht-Ordnung zu sprechen und den Trickster als „einsamen Erkunder der Nicht-Ordnung“ zu bezeichnen: Er ist fähig, die Nicht-Ordnung zu erfassen, indem er zwischen dem Kontinuum und dem Nicht-Kontinuum hinund her wandert. Dies kann nur als Unordnung (und Diskontinuum) wahrgenommen werden, weil die Perspektive des Erzählers von einer gegebenen Ordnung ausgeht (und von einem gegebenen Kontinuum), welchedieNicht-Ordnungdurch seineeigenen Kategorien definiert (Piasere2009). Die 
Funktion des Trickster wird fundamental wichtigin dem Moment, wo man dank seiner Anwesenheit „nicht nur eine Art von Ordnung als möglich definiert, sondern mehrere, auch alternative“, denn was für den Trickster wirklich von Bedeutung ist, ist „die Möglichkeit zur Wahl, sich außerhalb der Grenzen einer zwanghaften und unausweichlichen Notwendigkeit zu platzieren“ (Miceli 2000, 119).

Wie schon an anderer Stelle deutlich gemacht: Wenn das den Trickster ausmacht, ist dann die Erkundung der Nicht-Ordnung in einem Hin- und Her zwischen Kontinuum und Nicht-Kontinuum etwas anderesals derVersuch, dieIndizienfürdas Vorhandenseindes Unendlichen in derWeltzu erforschen? In den westlichen Traditionen hat man dieUnendlichkeit bekanntlich in Philosophie, Theologie und vor allem in der Mathematik gesucht. Die berühmte „Kontinuumshypothese“ von Georg Cantor, dievon unendlichen Mengen handelt, war der erste Punkt auf der 1900 von Hilbert aufgestellten Liste von mathematischen Problemen, die es zu lösen galt. Zu dieser Hypothese haben in den folgenden J ahrzehnten die größsten Logiker aller Zeiten, z.B. Göderl oder Coen, gearbeitet und gezeigt, dass sie weder bewiesen noch widerlegt werden kann. Die Kontinuumshypothese ist dabei nichts anderes als eine Variante des generellen menschlichen Problems, das von der Geordnetheit oder auch der NichtGeordnetheit des Universums handelt. Man sagt, die Mathematik sei die „Wissenschaft vom Unendlichen“ (Maor 1993, 6); die Ethnologen korrigieren: Mathematik ist die okzidentale Wissenschaft des Unendlichen. Giorgio Colli erklärt, dieBasis der modernen Mathemathiksei „derVersuch, das Unendliche mit endlichen Begriffen zu erklären“ (Colli 1998, 23). Brouwer, der Vordenker des mathematikphilosophischen Intuitionismus, sagte, die Intuition der mathematischen Grundlagen liege in dem Fakt, dass „Verknüpfung und Trennung, Stetigkeit und Un-Stetigkeit vereint sind“ (Brouwer 1964, 69). Wenn dies die Grundlagen der westlichen Wissenschaft von der Unendlichkeit sind, so ist die Untersuchung des Trickster ein gleichberechtigter Teil der Wissenschaft von der Unendlichkeit. Mir scheint, als gäbe es eine stillschweigende Übereinkunft, wonach die Erforschung der Unendlichkeit der okzidentalen Kultur vorbehalten sei. [1] Proust hat das bestätigt, indem er in seinem Roman eine Person erwähnte, die „am westlichen Ufer des Sees Victoria-Nyanza ein Werk über das Gefühl von Unendlichkeit" geschrieben habe (Proust 1988, 25) - die Ironie ist offensichtlich! Ich glaube, dass die Ethnologen mit der Figur des Trickster seit langer Zeit ein Beispiel vor Augen haben, wie ganz unterschiedliche Völker dieser Welt der Unendlichkeit in dieser Welt nachforschen.

Manchmal erscheint der Trickster als die Verkörperung der Unendlichkeit selbst, denn er definiert sich nach seinen eigenen Begrifflichkeiten: Radin bezeichnete den Trickster als eine Einheit
[1] Meines Wissens ist Jadran Mimica (1992) der einzige Anthropologe, der gezielte Forschungen über die Unendlichkeit in einer nicht-okzidentalen Kultur durchgeführt hat. Er hat das Nummerierungssystem der Iqwaye, einem Stamm in Neu-Guinea, untersucht. 
aus Verneinung und Bejahung; der italienische Mathematiker Paolo Zellini (1980, 236) beschrieb die Unendlichkeit als Union zweier unvereinbarer Seiten, der „reinen Affirmation und der reinen Negation".

\section{Die Zigeuner der italienischen Renaissance}

Vermutlich war es Paul Radin (1953), der als Erster die nordamerikanischen Trickster mit den Schelmen in Verbindung brachte. Karl Kerényi zufolge habe die moderne Schelmen-Literatur dieselbe Funktion wie die mythologischen Erzählungen über die Trickster, nämlich Ordnung und Unordnung zu vereinen (2006, 148-149). Er spricht in diesem Zusammenhang von der Mythologie der Winnebago als einer „Schelmenmythologie“. J edoch zitiert Kerényi nur Fälle aus europäischen Romanen und vernachlässigt ein literarisches Genre, in dem sich Figuren tummeln, die den Schelm antizipieren: die komödiantischen Heldengedichte. In drei italienischen heroischkomischen Gedichten, irgendwann zwischen dem 15. und 16. J ahrhundert verfasst, finden wir die ersten Figuren des Zigeuner-Trickster in der Weltliteratur. In diesen Figuren konstruiert sich, wenn man das so sagen darf, die "Zigeuner-Natur" in Etappen. Es handelt sich um Margutte, Brunello und Cingar. [2]

Margutte erscheint in „Morgante“ von Luigi Pulci, geschrieben zwischen 1461 und 1482. Er wird beschrieben als ein Wesen von schwarzer Haut, mit „,komischen, schrecklichen, hässlichen“ Gliedern: Er hätte ein Riese werden sollen, hat aber entschieden, sein Wachstum zu unterbrechen und blieb ein Halb-Riese. Dieses Monströse - weder normal noch Riese - dieses hybride Aussehen, darf nicht von seiner kulturellen Monströsität getrennt werden. Er kommt an verschiedenen Stellen des Gedichts vor:

1) Margutte ist ein Sarrasin, ein Araber.

2) Margutte ist weder Sarrasin noch Christ.

3) Margutte ist zu einer Hälfte Sarrasin und zur anderen Hälfte Christ.

4) Margutte ist eine Fusion aus Sarrasins und Christen.

Diese ethnisch-religiöse Monströsität scheint die Ursache aller Laster und der Unehrlichkeit von Margutte zu sein. Er ist ein Vatermörder, ein Schlemmer, luxussüchtig, ein Spieler, Dieb, Fälscher,
[2] Ich nehme hier Überlegungen auf, die ich in Piasere (2006) und Campigotto/ Piasere (1990) dargelegt habe. 
meineidig, Betrüger, blasphemisch, Streithahn, unverschämt, dreckig etc. Nach seinem Tod wird er Diener des Teufels. Die Monströsität von Margutte kommt entweder von seiner hybriden Herkunft - er ist der Sohn einer griechischen Ordensfrau und eines türkischen Hodscha; oder von dem Fakt, dass man ihn nirgendwo richtig einordnen kann - weder Christ noch Sarrasin oder beides; oder durch seine opportunistischen Entscheidungen, denn er verkündet, Recht und Ordnung zu ändern, wie es ihm beliebt.

Das Gedicht von Pulci ist das erste italienische Werk, das Zigeuner erwähnt. Siekommen auch in der Episode von Margutte vor, aber Margutte ist kein Zigeuner. Dennoch wird anhand der anderen Figuren klar, dass es sich bei ihm um einen „Prä-Zigeuner“ handelt.

Zahlreiche Autoren haben Margutte mit Brunello in Verbindung gebracht, der Figur, die in Maria Matteo Boiardos „Orlando innamorato“ (Der verliebte Roland) von 1483 auftaucht. Es scheint mir jedoch, dass niemand die Spurverfolgthat, dieich hiervorschlage. Wiebei Marguttewird bei Brunello im Allgemeinen unterstrichen, er gehöre zu „einer Klasse“, einer Klasse, welche die Historiker der Frühen Neuzeit „Randgruppe“ nennen. Ich möchte hier hingegen seine kulturellen Charakteristika analysieren. Margutteist ein Halb-Riese, Brunello (wörtlich: „Heller Schwarzer“) ist ein Halb-Zwerg, ein Schurke und König der Diebe. In einem Teil des Gedichts tollt er raubend umher, bestiehlt die größten Könige, Prinzessinnen und Paladins, ja er stiehlt Roland sogar seinen berühmten Umhang und sein berühmtes Schwert! Er bedroht die Welt mit „kosmischen“ Diebstählen, wie den Diebstahl des Mondes, den Diebstahl der Gabel des Teufels, den Diebstahl des Klangs der Glocken und der Entführung des Papstes usw. Am Ende wird er König von Tingitane, das heißt dem westlichen Ende des nördlichen Afrika, der Region des heutigen Marokko, wo sich Tanger befindet. Diese Beförderung lässt uns verstehen, dass Brunello ein Crypto-Zigeuner ist, denn Boiardo hat in Ferrara studiert und dort sagte man zu jener Zeit, Zigeuner seien tingitane-Mauren, die durch Europa irrten, nachdem sie von ihrem Land durch die Sarrasins verjagt worden seien.

Wenn es auch der Rekonstruktion bedarf, um die Zigeuner-Identität von Brunello zu zeigen, so ist es viel einfacher, seine „Trickster-Praktiken“ nachzuweisen. Ich beziehe mich dabei nicht auf sein unsoziales Verhalten (z. B. seine Diebstähle), sondern auf sein „politisches“ Verhalten. Brunello ist ein Entweiher der politischen und moralischen Ordnung, ein Entweiher der soziokulturellen Ordnung, noch bevor er ein Dieb ist. Seine Entweihung ist keine totale Ablehnung, sondern eine teilweise Entfernung: Brunello lebt immer über der Aktion, in die er gerade verstrickt 
ist. Zwischen ihm und der Gesellschaft herrscht immer eine Lücke, eine Entfernung, die er mit sinnstiftender Ironie kompensiert. Mit der Entweihung der Hierarchien und der Sicherheit der anderen schafft er seine eigene Welt. Ein Beispiel für diese Kultur der Entweihung à la Brunello ist die Episode, in der er das Schwert der indischen Königin Marphise stiehlt. Diese verfolgt Brunello über einige Gesänge des Gedichtes und muss sich unglaubliche Schmähungen anhören. Er gibt ihr zu verstehen, was er von ihr hält, von ihr und ihrem Rang - und zwar durch Gesten, die ihresgleichen suchen, er hebt seine Kleidung und zeigt ihr sein Hinterteil etc. Nur diejenigen, die sich - ganz nach Montaigne - dessen bewusst sind, dass man, egal wie hoch man platziert sein mag, doch immer nur auf seinem Hintern sitzt, können diesen ohne Scham einer Königin zeigen. Die Unverschämtheit Brunellos beschränkt sich nicht nur auf irdische Hierarchien, er verspottet auch die Lehren des Evangeliums. Als die sarrazenische Armee in Frankreich einfällt, hisst Brunello als König von Tingitanien sein Banner: eine Gans auf rotem Grund, die ein Ei legt. Warum eine Gans als Symbol? Brunello wollte zeigen, dass er, der neue König, in Realität die ältesten Wurzeln aller hatte, im Anbeginn der Zeit, als man nicht wusste, wer zuerst geboren wurde: das Huhn oder das Ei. Er erklärte, das Evangelium würde lehren: „Am Anfang war die Gans“. Hierbei handelt sich um einen blasphemischen Streich, der auf dem J ohannes-Evangelium $(1,2)$ beruht: „Hoc erat in principio apud Deum“ - hoc ist ein Pronom, das das Wort ersetzt, das Brunello hier aber im italienischen Dialekt als oc, Gans, ausgibt: Es ist nicht mehr das Wort, sondern „die Gans, die am Anfang bei Gott war"!

Die „Zigeunerisierung“ dieser komischen Helden geschieht in aller Vollständigkeit mit der von Teofilo Folengo geschaffenen Trickster-Figur, diepräziserweise auch den Namen Cingar, „Zigeuner“, trägt. Cingar kommt in mehreren Werken dieses Autors vor, aber vor allem in „Baldus“, einem in makkaronischem Latein [„Küchenlatein“, A.d.Ü.] verfassten Gedicht, das 1517 veröffentlicht wurde. Es gab eine schöne Debatte zwischen den Kritikern um den Einfluss, den „Baldus“ auf das berühmte Werk „Gargantua et Pantagruel“ von François Rabelais hatte. Radin und Kerényi zitieren ihn als Vorreiter in der Schaffung von Trickster-Figuren, andere sahen in Cingar die Figur, die Rabelais zur Schaffung von Panurge inspirierte (Tetel 1963). So macht Folengo aus Cingar-der-Zigeuner einen direkten Nachfahren von Margutte-der-Vor-Zigeuner, indem er ihn von seinem Blut („Caos del Triperuno“" „Narratio“, „Selva II“) und seinem Samen (Baldus, IV, 129) abstammen lässt. Cingar stammt vom Blut und Samen eines Räubers und ist selbst der größte Räuber: 
Cingar scampasoga, cimarostus, salsa diabli, accortusque ladro, semper truffare paratus, in facia scarnus, reliquo sed corpore nervax, praestus in andatu, parlatu, praestus in actu, semper habens testam nudam penitusque tosatam.

Praticus ad beffas, truffas, sardasque soiasque,

deque suo vultu faciens plus mille visazzos,

et simulans varias sguerzo cum lumine morfas. (IV, 82-90) [3]

Verglichen mit Margutte und Brunello hat sich das Äußere von Cingar stabilisiert. Diese Normalität überdeckt jedoch nicht völlig eine groteske Physiognomie mit seinem Gesicht (faciens plus mille visazzos, et simulans varias sguerzo cum lumine morfas). Die physische Monströsität weicht der physischen Groteske, und die groteske Darstellung von Gesichtern der Zigeuner-Männer beschränkt sich nicht nur auf die Literatur. Ich berichte hier in einer Parenthese von einem wenig bekannten Fall, obwohl er in Verbindung mit dem Genie Leonardo da Vinci steht, einem Zeitgenossen Folengos. Verschiedene Kunsthistoriker haben geglaubt, in dem Gesicht, das in Abbildung 1 zu sehen ist, den „Scaramuccia capitano de' Zingani“ zu erkennen. Es handelt sich um eine der Zeichnungen da Vincis, die verloren gegangen sind, und von der Giorgio Vasari in Le vite de' più eccellenti pittori, scultori, e architettori (1567) spricht. Die Skizze des Gesichts wird in der Christ Church Library von Oxford aufbewahrt. In den Da-Vinci-Katalogen wird sie unterschiedlich betitelt, zum Beispiel als „Portrait of a Gypsy in profi oder „Grotesque head of a man“ etc. (Hamill 1942). Auch wenn die Identifikation der Zeichnung nicht vollständig gesichert ist, so haben es doch mehrere Autoren des J ahrhunderts leicht und „normal“ gefunden, so als wäre es selbstverständlich, dass man ein groteskes Gesicht mit einem Zigeuner-Gesichtin Verbindung bringt. Dies hat unter anderem auch Ernst Gombrich bemerkt (1986).

Ich möchte daran erinnern, dass ein groteskes oder monströses Aussehen in Verbindung mit gewiefter Intelligenz von Koepping (1985) als ein transkulturelles Merkmal des Trickster ausgemacht wurde, es verweise auf den Umsturz der Ordnung, den der Trickster metaphorisch verkörpert. Der Autor unterstreicht, dass die "two facets of the universal figure of anomaly and ambiguity, those of crooked thinking and of a grotesquely extended body“ der paradigmatische Ausdruck „of the experience and perception of absurdity“ seien. Ein schlauer Geist und ein deformierter Körper sind nicht immer gemeinsam vorhanden, aber sie treffen auf „most of the trickster figures in narrations
[3] Übersetzung: Cingar, wildes Tier der Potenz, Blume des Halunken, Eintopf des Teufels, bauernschlau, Dieb, immer bereit zum Betrügen, ein scharfes Gesicht, sein restlicher Körper in gutem Zustand, bereit zu marschieren, bereit zu sprechen, bereit zu handeln, der Kopf immer nackt und rasiert, Experte im Ohrfeigen, in schnippischen Bemerkungen, im Betrügen, mit 1000 Gesichtern und verschiedenen Minen

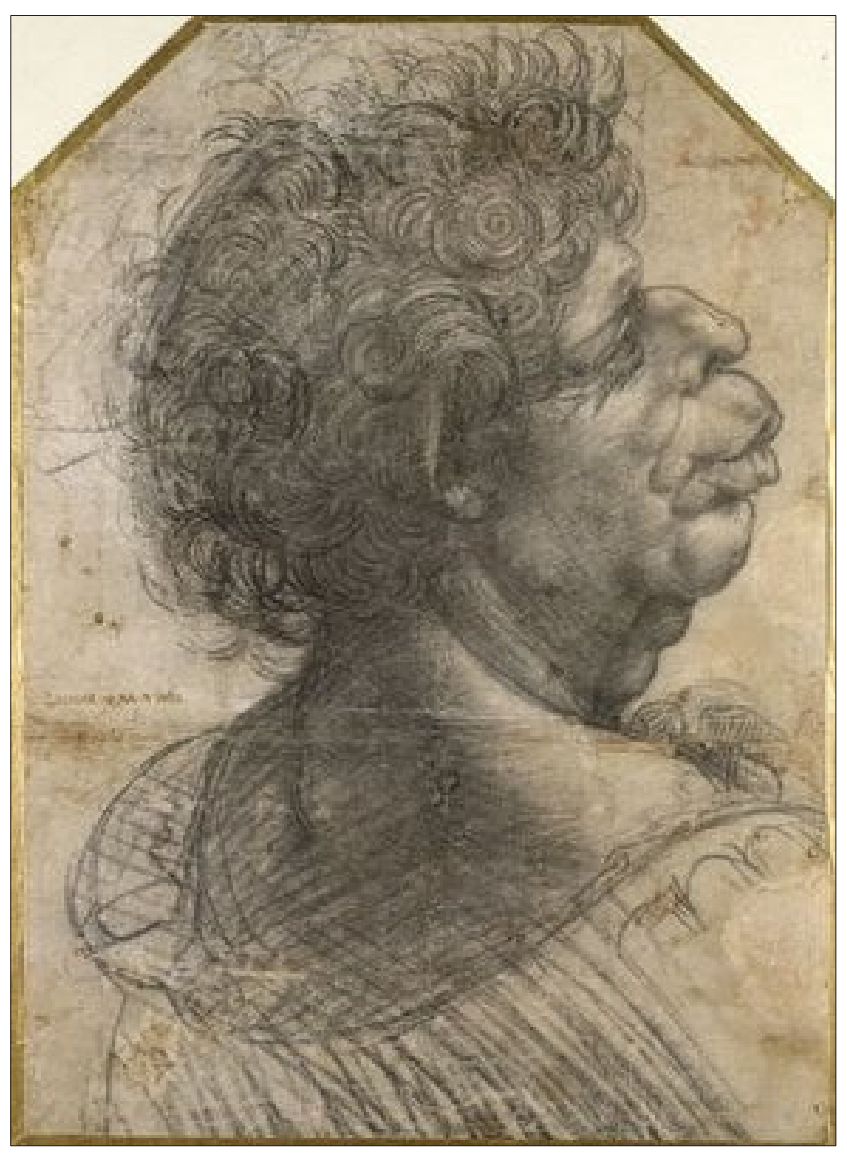

[Abb. 1] 
as well as the secular extensions of it“ $(1985,196)$ zu. Und die Figuren Margutte, Brunello und Cingar passen perfekt in dieses Fresko à la Bakthin. Diejenigen, die aus dem Weltbild religiöser Dichotomie der Epoche fallen, werden mit einer deformierten religiösen Identität dargestellt: Margutte als verdorbener Sarrasin, Brunello als frecher Sarrasin und Cingar als degenerierter Christ. Die Verbannung Cingars aus Mantua, wo er lebte, erinnert an die Erlasse zur Verbannung der cingari, der Zigeuner, welche die italienischen Staaten der Epoche in Hülle und Fülle zu veröffentlichten begonnen hatten:

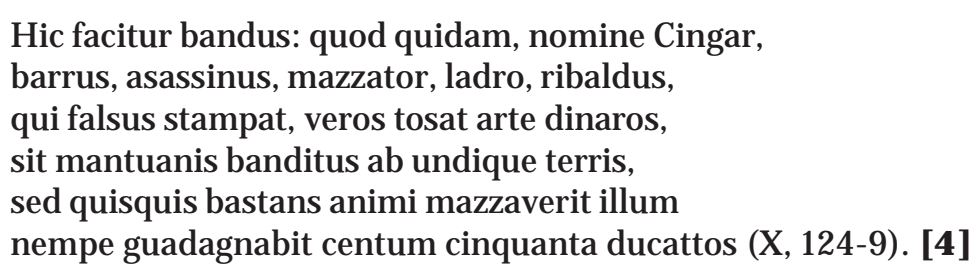

Mit dieser Schrift gegen den Cingar sehen wir, wie die souveräne Herrschaft sich gegen jene erhebt, die es wagen, die Grenzen der die Herrschaft legitimierenden Taxonomie zu stören oder sich einzumischen. Wie Ballinger schrieb, „Euro-American, Christian culture survives by clear definition of categories. Indeed, the entire Christian conception of history - individual and cosmic - as a struggle against the evil empire demands such definition. As Stanley Diamond points out, two works central to the Euro-American and Christian world view, the Book of J ob and Plato's Republic, are bent 'upon denying human ambivalence and social ambiguity'“ (1991, 27-28). Der gefährliche Charakter der Zigeuner ist vollständig vereinigt in der Art und Weise, wie sie in einer lateinischen Version der "Cosmographie“ von Sebastian Münster dargestellt sind, dem berühmten deutschen Gelehrten und Zeitgenossen von Folengo. Er beschreibt sie als „gentiles christiani“, als heidnische Christen - die Vereinigung der Gegensätze! Darauf werde ich gleich noch zurückkommen.

Es ist wichtig hervorzuheben, dass in den komödiantischen Heldengedichten eine klare Trennung zwischen dem Zigeuner und der Zigeunerin gemacht wird. Was die Zigeuner-Familie betrifft: sie existiert ganz einfach nicht. Wie es sich für einen Trickster gehört, ist Margutte der „Vor-Zigeuner" ein einsamer Vagabund und außerdem noch ein Vatermörder; Brunello der „Crypto-Zigeuner" hat gar keine engen Verwandten; Cingar ist ein Gattinnenmörder und seine Frau gehört zu den Hexen der Hölle. Die Zigeunerin spielt keine Rolle in der Welt der Helden- und
[4] Übersetzung: Hiermit wird verkündet, dass ein sogenannter Cingar, Mörder, Dieb, Bösewicht, der Falschgeld hergestellt hat und die Ordnung stört, aus Mantua verbannt wird und dass jeder, der ihn tötet, mit 150 Dukaten belohnt wird. 
Ritterromane. Sie ist literarisch in den Gesängen und Karnevalsspektakeln nicht präsent, taucht dafür aber vor allem im 17. J ahrhundert in Theaterstücken mit dem Namen Zingaresche (vgl. Piasere 2006) auf.

Wir interessieren uns hier für einige Zingaresche aus dem 16. J ahrhundert, um einen linguistischen Aspekt zu betrachten. Die Sprachen, die Margutte, Brunello und Cingar benutzten, waren das Toskanische und makkaronisches Latein - also die Sprachen, in denen die Werke verfasst waren oder in denen sie zum ersten Mal erschienen. Alle drei sprechen auch die lingua zerga, den J argon der Kriminellen. Der Gebrauch des zergo unterstreicht natürlich ihre soziale Außenseiterschaft. Die Zigeunerinnen in den Zingaresche sprechen keinen solchen Dialekt. Ihre Ausdrucksweise hat zwar die Tendenz, sich von jener der anderen Figuren abzugrenzen, jedoch ohne dass auf einen J argon zurückgegriffen wird, wie er den Männern in den Mund gelegt wurde. Dies zeigt einerseits, dass Zigeuner und J argon nicht automatisch zusammengehören, anderseits aber auch - und darauf hat der Philosoph Giorgio Agamben mit scharfem Blick hingewiesen (1996) - dass die virtuelle Gleichzeitigkeit ihrer Erscheinung in den Diskursen der Renaissance daraus eine Treibmine für unser Verständnis von „Volk“ und „Sprache“ macht. Und diese beiden Konzeptionen sind grundlegend für unsere Idee vom Nationalstaat, die wir seitdem konstruiert haben. Die sprachlichen Fähigkeiten der Zigeuner-Frauen sind umfangreich und es gibt von Komödie zu Komödie viele Möglichkeiten:

1) DieZigeunerin spricht dieselbe Sprache wie die anderen Figuren (obwohl dies im 17.J ahrhundert ein gehobenes Italienisch ist, das auch weiter vorherrschen wird).

2) Die Zigeunerin spricht die Sprache des Komödien-Autors, allerdings nur gebrochen.

3) Die Zigeunerin spricht eine Sprache mit südländischem Akzent (im 16. Jahrhundert finden sich die Zingaresche vor allem in Nord- und Zentralitalien).

4) Die Zigeunerin ist eine Wahrsagerin und spricht in Reimen.

5) Die Zigeunerin spricht eine unverständliche Fremdsprache (die linguistische Analyse zeigt, dass es sich um Arabisch oder Romani handelt). 
Wie man sieht, gibt es den ausdrücklichen Willen, die Sprache der Zigeuner in den Vordergrund zu rücken. So unterstreicht eine Autorin, dass man „den Kosmopolitismus der Figuren der Zingaresche bewahren muss; sie sind nicht nur aus dem Orient, sondern auch aus dem Okzident, wie im Fall der Figur des Franzosen, die oft ins Lächerliche gezogen wird“ (Greco 1976, 525). Tatsächlich werden die Zingaresche des 16. J ahrhunderts in die mehrsprachigen Theaterproduktionen integriert, die ihre größten Erfolge mit der Commedia dell'Arte und in den Ridicolose-Komödien feiern. Dies liegt einmal an der weiten Verbreitung der Zingaresche, die dazu führte, dass seit Cesare Ripas Iconologia (1593) während des ganzen Barock die Zigeunerin das Symbol der Komödie schlechthin wurde. Gibt es darüber hinaus auch einen kulturellen Grund? Ich versuche, darauf mit Zitaten von Leach und Sperber zu antworten:

- „Alle Menschen, aus aller Welt, verhalten sich infolge ihrer kulturellen Prägungen so, als gehörten sie zu vielen verschiedenen Arten. “ [5] (Leach 1980, 365)

- „Die Ausstellungen mit wilden Tieren [Zoos, Rummel, Zirkus, Delphinarium] dienen nicht nur der Befriedigung simpler Neugier: Neugier ist niemals simpel. Durch diese kulturellen Institutionen sollen die Einwohner der Städte, insbesondere die Kinder, über die Beziehung der Arten untereinander und die Beziehung der Tierwelt zu den Menschen nachdenken. " [6] (Sperber 1975, 28)

Die Zingaresca des 16. J ahrhunderts war eine Art „Zoo“-Komödie, in der die lokalen Bewohner Nord- und Zentralitaliens als verschiedene Menschen-„Arten“ dargestellt und miteinander verglichen wurden. Im Rahmen dieser ethnischen Klassifizierung hat die Zigeunerin symbolischen Wert, denn ihre taxonomische Identifikation wurde unter „Normalitätskriterien“ diskutiert:

1) Sie ist Teil der Menschheit, auch wenn sie in Höhlen und Hütten lebt.

2) Sie kommt aus einem muslimischen - barbarischen - Land oder von „weither“, aber sie ist dennoch Christin.
[5] „Sous l'effet de leurs inhibitions culturelles, les hommes, tous tant qu'ils sont et partout de par le monde, se comportent comme s'ils étaient les membres de nombreuses espèces différentes."

[6] „Les exhibitions d’animaux sauvages [zoos, foires, cirques, delphinarium] ne sont pas là pour satisfaire une simple curiosité: la curiosité n'est jamais simple. Ce sont des institutions culturelles par le moyen desquelles les habitants des villes, et particulièrement les enfants, sont invités à penser symboliquement le rapport des espèces entre elles et le rapport de la faune à l'humanité." 
Das Gelächter, das sie bei den Zuschauern auslöste, korrespondiert mit der sozialen Verurteilung, welche die Zigeuner im täglichen Leben ertragen mussten. Aus dieser Sicht hilft eine Analyse der Beschreibung der „Christen-Heiden“ durch Sebastian Münster (1559, 267-268), um zu verstehen, worin die große Sünde der Zigeuner bestand. Ihre Sünde war das Praktizieren des Kosmopolitanismus. Sie „reden wie Hunde“ (canino ritu), und zwar nicht, weil sie des Sprechens nicht mächtig sind (wie man es von den „Barbaren“ der Antike behauptete), sondern ganz im Gegenteil, weil sie alle Sprachen beherrschen: „Eine Vielzahl [...], die in den Sprachen aller Menschen spricht“ (omnia perita linguarum) - ein Anti-Babel, dass aus einer zufälligen Mischungund ohne Selektion entsteht: „Sie empfangen alle möglichen Leute aus allen Provinzen“ (recipiunt passim et viros et faeminas [...]in cunctis provinciis). Ein Anti-Babel mit seinem eigenen „Barbarentum“, das den allgegenwärtigen Gegensatz zwischen dem Einen und der Vielzahl aufhebt (sie sprechen eine Sprache/ sie sprechen alle Sprachen). Eine Mischung, die, so könnte man sagen, über das Kulturelle hinausgeht. Sie gebären ihre Kinder auf der Wanderschaft (in peregrinatione), überall und nirgends, außerhalb der nationalen Ordnungen, in ständiger, nicht zu fassender Bewegung durch alle Kulturen und die Länder Europas hindurch, wo die Fortbewegung auf dem Territorium durch Pässe und Ähnliches überreglementiert war. Wie Münster es so gut dargestellt hat, erschienen diese Panglossie und dieser Pankulturalismus monströs, und wir finden diese Eigenschaften mit verschiedenen Aspekten bei allen bereits erwähnten literarischen Figuren. Es hilft uns, die Zigeunerin als Symbol der Commedia von Cesare Ripa zu verstehen, als Symbol „aller Zustände und aller menschlichen Eigenschaften“ (Münster 1593, 46), das sich außerhalb jedes Verständnis' befindet. Die Zigeunerin repräsentiert ein monströses metakulturelles Niveau, das jeden „Lokalismus“ [localisme] überschreitet und nur als Ausnahmezustand existieren kann.

Man könnte sagen, dass die Zigeunerin ein Mana-Symbol ist, ein Symbol, das alle kulturellen Bedeutungen vereint und gleichzeitig keine einzige. Ein deutlicher Hinweis ist zum Beispiel, dass Münster das "Sprechen wie Hunde“ mit Religionslosigkeit in Verbindung bringt - eine umgekehrt konstruierte symbolische Handlung. Erfolgte die Abschaffung des Einen und der Vielzahl vorher durch den Besitz einer eigenen Sprache oder durch gleichzeitiges Praktizieren aller Sprachen, erfolgt sie hier durch das Vorhandensein einer Religion (der christlichen: sie taufen ihre Kinder) und dem Fehlen jeglicher Religion. Hier hilft die Zigeunerin als symbolische Konstruktion, die Worte Münsters zu deuten, der sich weniger auf das Fehlen einer Religion 
als vielmehr auf das Fehlen einer Religionswahl bezieht. Tatsächlich scheint die Zigeunerin alle Religionen zu praktizieren:

1) In den Zingaresche ersetzt die Zigeunerin die Sibylle, die Prophetin, und genau wie sie tritt sie als Repräsentantin auf Erden des gesamten antiken Pantheons auf.

2) Obwohl sie christlich ist, wird die Zigeunerin oft mit den Juden in Verbindung gebracht. Entweder ist sie Nachkomme jüdischer Eltern oder sie wird klar als J üdin identifiziert. Einige Zingaresche heißen einfach nur „la Zingara giudìa“, diejüdische Zigeunerin.

3) Im 16. J ahrhundert stellen viele Bilder die Heilige J ungfrau als Zigeunerin dar. Ich möchte nur zwei Beispiele erwähnen: Zum einen das der „La Zingarella“, einer berühmten J ungfrau, die in Wien aufbewahrt wird und deren Zuordnung ungewiss ist (Giorgione oder Tizian) [Abb. 2]. Zum anderen „Das Gewitter“ [Abb. 3] von Giorgione, auf dem eine Frau zu sehen ist, die im 16. J ahrhundert entweder als J ungfrau oder als Zigeunerin identifiziert wurde (vgl. Partington 1956).

Hier also findet sich das Mana-Symbol: Annullierung von Polytheismus und Monotheismus sowohl seitens des Gottesmordes (Zigeunerin = Jude) als auch seitens der göttlichen Entstehung (Zigeunerin = J ungfrau). Dennoch ist die Übersetzung der Zigeunerin niemals „Vollständig“ oder „sicher“. Eben konstatierte ich, die Zigeunerin sei Symbol der Abwesenheit von Auswahl, aber das stimmt nicht ganz. Die Zigeunerin ist in Wahrheit der Signifikant einer Auswahl, die begann und dann verschwand, eine missglückte Auswahl: So wie Margutte ein missglückter Riese ist und Brunello ein missglückter Zwerg, so ist die Zigeunerin eine Christin und gleichzeitig auch alles andere. Man kann die Bedeutung der Zigeunerin nicht mit richtig/falsch oder alles oder nichts entschlüsseln, sondern nur mit der Logik des fuzzy, des „ein bisschen“, des alles und nichts. Im frühneuzeitlichen Italien bestand das Monströse, das Unordentliche der Zigeuner darin, dass sie als kulturelle Krüppel gedacht wurden - von denjenigen, die sich selbst nicht als Zigeuner verstanden: ein bisschen von allem/von jedem ein bisschen, die Verkrüppelung ist Fehlen und Vorhandensein einer Identität. Radin sagte, der Trickster sei „alles geworden und geblieben, in den Augen aller“ (2006, 133).

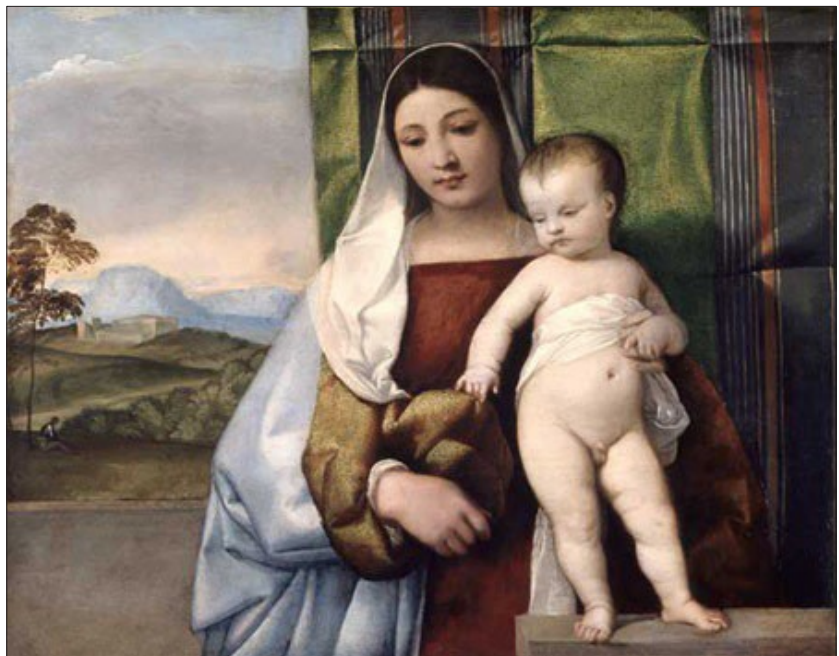

[Abb. 2]

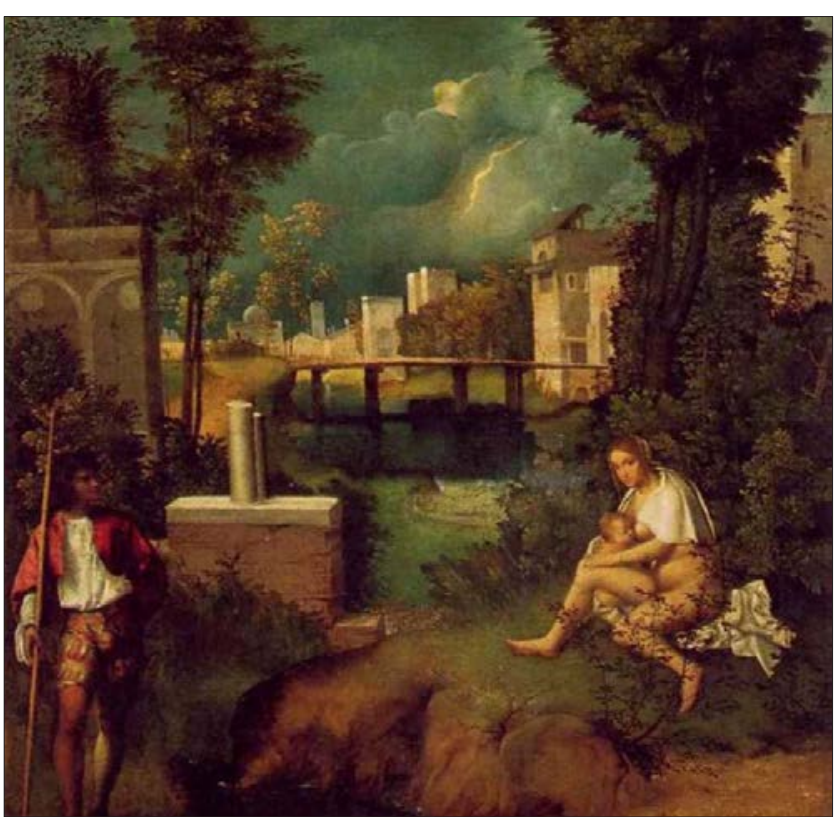

[Abb. 3] 


\section{Von der italienischen Renaissance zur rumänischen Illuminationsphilosophie}

Ion Budai-Deleanu, ein transsilvanischer Dichter, der zwischen dem 18. und 19. Jahrhundert gelebt hat, hat das unglaublichste Buch derWeltliteratur geschrieben, dessen Protagonisten Zigeuner sind: „Tiganiada“. Es handelt sich auch hier um ein komödiantisches Heldengedicht, eines der ersten großen Werke der rumänischen Literatur, und beschreibt einen Ausschnitt aus dem Kampf des Woiwoden Vlad III, genannt der Pfähler (und berühmt geworden unter dem Namen Dracula), gegen den Vormarsch der Osmanen. Wir befinden uns im Fürstentum der Walachei, im 15. J ahrhundert, wo Himmelsengel an der Seite der christlichen Armee gegen Teufel kämpfen, die aus der Hölle gestiegen sind, um der türkischen Armee zu helfen. Vor diesem „kosmischen“ Hintergrund sehen wir die außergewöhnliche Pazifisten-Armee der Zigeuner. Sie werden vom walachischen Woiwoden als Armee zusammengestellt und, falls sie sich als siegreich gegen die Türken erweisen, verspricht er ihnen ein Dorf, in dem sie leben könnten, Nomadentum und Diaspora endlich hinter sich lassend. Die Zigeuner-Armee wird zum großen Kollektiv-Trickster, in dem sich Individuen und Gruppen zusammenschließen, um die absurdesten Situationen zu schaffen. Die Intention des Autors ist ganz klar eine allegorische: Der Dichter wollte durch die Zigeuner die Verfassung der Rumänen in der ersten Hälfte des 19. J ahrhunderts zeigen: zu einfältig, um sich vom J och der Fremdherrschaft zu befreien (Türken und Habsburger). Dabei bedient er sich eines ethnographischen Realismus, der dem ganzen Gedicht eine besondere Färbung verleiht.

Die Zusammensetzung der Armee stimmt mit den sozialen Charakteristika der Roma der Region überein (insbesondere mit seiner anpassungsfähigeren transsilvanischen Variante): Die Berufsgruppen dienen als Grundlage der sozialen Organisation, insbesondere in den Beziehungen zum Staat (Piasere 2005). DieArmee besteht aus sieben Cete, Gruppen: den Ciurari (Siebhersteller), den Argintari oder Zlătari (Vergoldern), den Căldărari (Kesselschmieden), den Fierrari (Schmieden), den Lingurari (Holzhandwerker), den Aurari (Goldsuchern) und den Lăieschi oder Goleschi (arme Vagabunden). J ede Gruppe hat ihren Anführer, aber das Oberhaupt der Argintari, Parpangel, ist theoretisch auch der Anführer der gesamten Armee, deren Hierarchisierung nicht ganz sicher ist. An der Spitze stehen anscheinend die Aurari und die Argintari, ganz unten die Ciurari und vor allem die Lăieschi. Insgesamt scheint dies ganz gut damit zu korrespondieren, wie die Nicht-Zigeuner 
der Region die Zigeuner sahen und für jede Gruppe Stereotype schufen. Auf dieser realistischen Grundlage basieren die Ereignisse des Gedichts, beginnend mit dem ulkigen Defilee der sieben Cete vor Prinz Vlad. J ede Gruppe hebt ihre verrückten Standarten und zeigt ihre Waffen. Die Standarte der Argintari zum Beispiel, zu denen auch der Zigeuner-Woiwode Parpangel zählt, ist ein Rabe mit ausgestreckten Flügeln. Hierbei handelt es sich um ein pseudo-totemistisches Symbol, denn in der Region wird der Zigeuner gerade Cioăra genannt, Rabe.

Die Reise in das Dorf, in das die Zigeuner-Armee ziehen sollte, wird zu einer Reise ins Absurde: Zuerst wollen die Zigeuner dem Prinzen einen Botschafter schicken, um ihn zu bitten, die Wegeslänge zu verkürzen, die ins Dorf führt. Dann schlägt jemand die ausgefallensten Lösungen vor, wie man am Besten fliehen könnte, sollten die Türken wirklich angreifen. Der Teufel kommt hinzu und entführt Romica, die Verlobte von Parpangel, um den Marsch der Zigeuner zu verlangsamen. Das führt sogar so weit, dass Parpangel sich in Wäldern und verzauberten Orten auf die Suche nach ihr macht. Seine Mutter ist Zauberin und hilft ihm an einer Stelle mit ihrer Magie. Und schließlich, um die Tapferkeit der Zigeuner zu testen, verkleidet Vlad seine eigenen Soldaten als Türken und greift die Zigeuner an: Sie unterwerfen sich sofort ohne Kampf! Die List wird aufgedeckt und Vlad zieht davon. Die Zigeuner nehmen ihre Reise wieder auf, werden aber von den Türken angegriffen, diesmal von den richtigen. Weil sie sich sicher sind, dass es sich erneut um Vlads Armee handelt, verteidigen sie sich und kämpfen wie die Löwen. Als Vlad dann später die Türken angreift, erstarren die Zigeuner vor Furcht und Schrecken bei dem Gedanken, sie ihrerseits angreifen zu müssen. Sie beschließen deshalb, sich mit geschlossenen Augen auf sie zu werfen, um die Angst so lange fern zu halten, bis der Tod kommt. Statt gegen die Türken kämpfen sie dann allerdings gegen eine Kuhherde. Die Walachen besiegen die Türken letztendlich, aber es sind die Zigeuner, die in ihr Lager einfallen und alles mitnehmen, was sie finden - besonders Unmengen an Nahrung. Als sie endlich im versprochenen Dorf ankommen, feiern sie die Hochzeit von Parpangel, der Romica in der Zwischenzeit wiedergefunden hat, mit einem üppigen Festmahl. Das passt zu den anderen Ereignissen des Gedichts, bei denen die Zigeuner immer auf der Suche nach Nahrung zu sein scheinen. Selbst das Paradies, in dem Parpangel im Laufe seiner Abenteuer landet, wird wie ein Schlaraffenland beschrieben, wo alles, auch die Flüsse, die Berge und die Wälder aus essbaren Köstlichkeiten bestehen. Die Zigeuner-Armee verliert sich ständig in Diskussionen, in denen alle reden und niemand zuhört. Die bekannteste Diskussion ist ohne Zweifel die, wo sie sich ohne Ende darüber 
streiten, wie ihr ideales politisches System aussehen soll, wenn sie einmal eine Heimat haben. Sie zählen die zu besetzenden Posten auf, wobei ziemlich genau das zu Budai-Deleanus Zeit herrschende walachische Feudalsystem abgebildet wird. Schnell breitet sich die Diskussion dann auf die großen politischen Fragen aus, nämlich welches Regierungssystem gewählt werden sollte. Man ernennt eine Kommission gelehrter Zigeuner, die Vorschläge machen sollen. In endlosen Reden erklärt ein Gelehrter die Monarchie zur besten Regierungsform, ein anderer die Demokratie. Ein dritter plädiert für ein hybrides System, das er Demo-Aristo-Monarchie nennt. Dieser Vorschlag scheint die meiste Zustimmung zu erhalten, als das Zigeuner-Volk in die Versammlung eindringt und alle gelehrten Diskussionen verhöhnt. Es kommt zu einem allgemeinen Handgemenge, bei dem es Tote und Verletzte gibt und das an einen beginnenden Klassenkampf erinnert. Dieärmsten Cete kämpfen gegen die angeseheneren: Die Cuirari schließen sich mit den Lăieschi gegen die Zlatari zusammen, die Căldărari kämpfen gegen dieAurari. Am Ende verstreuen sich die Zigeuner-Gruppen und jeder zieht allein weiter, während Vlad, obwohl er gewonnen hat, sich der Entscheidung des Himmels unterwerfen und sich den Türken geschlagen geben muss.

Die Zigeuner der „Tiganiada“ wurden von einer Kommentatorin als „Kreaturen des unendlichen Chaos, betrachtet durch die Variabilität eines Kaleidoskop“ bezeichnet, in einem Gedicht, wo „ein Universum beschrieben wird, in dem eine fröhliche Mischung zwischen dem Natürlichen und dem Übernatürlichen herrscht, zwischen dem Realen und der Vorstellungskraft; die Darstellung einer verkehrten Welt, die das Bild einer Menge überraschender Metamorphosen und außergewöhnlicher Verwandlungen bietet" (Senatore 2006, 124, 120). Der Einfl der komödiantischen Heldengedichte klassischer (wie beispielsweise der Froschmäusekrieg von Homer) und ausländischer Autoren (unter anderem Teofilo Folengo) wird vom Autor selbst eingestanden und von den Kritikern bemerkt. J edoch handelt es sich hier, und das soll unterstrichen werden, anders als in der italienischen Renaissance nicht nur um die Konstruktion eines Gelehrten. Die französische Kulturwissenschaftlerin Micheline Lebarbier (1980, 1989, 1991) hat in einer Reihe von Studien gezeigt, dass die Zigeuner bei Budai-Deleanu einerseits das Bild vom Zigeuner widerspiegeln, das bis heute in der rumänischen Populärkultur und besonders den spaßhaften Fabeln kolportiert wird, dass der Zigeuner andererseits aber in der mündlichen Überlieferung oft den Păcală ersetzt. Der Name dieser bekannten Figur kommt vom Verb a păcăli, was soviel wie „einen Streich spielen“ bedeutet. Anders ausgedrückt: Der Păcală ist nichts anderes als ein lokaler Wakdjũnga, ein rumänischer 
Trickster. Man kann Zigeuner und Păcală manchmal durcheinander bringen, einer ist manchmal das Doppel des anderen und zeigt sich überall da, wo List und Unsinn vorkommen. Lebarbier zufolge zeichnet sowohl den Zigeuner bei Budai-Deleanu als auch jenen in der Populärkultur eine lächerliche Albernheit aus, die besonders zum Vorschein kommt, wenn er „versucht, soziale Regeln zu imitieren oder sich in die Gesellschaft zu integrieren. Diese Erzählungen berichten von der Ohnmacht der Zigeuner“ (Lebarbier 1991, 192). Der Wunsch des Zigeuners, „zu sein wie die anderen“ endet in Absurdität: Deshalb „zeigen diese Texte seine ungeschickten Versuche, sich zu integrieren und die abweisende Haltung der Gesellschaft“ (ebd.). Der rumänische Autor Mihai Rezvan hat darauf aufmerksam gemacht, dass es zwar richtig ist, dass Budai-Deleanu die Zigeuner Anfang des 19. J ahrhunderts als historische Allegorie nutzte, dass aber die weite Verbreitung der „Tiganiada“ vor allem in den rumänischen Schulbüchern des 20. J ahrhunderts dazu beigetragen hat, die „Tiganiada“ „nach und nach zum Synonym des ,Zigeunertums’ zu machen: eine abstrakte Referenz, ein karikaturales, also deformiertes Bild einer Gruppe von Menschen“ (Rezvan 1985, 122). Unserer Meinung nach resultiert diese Deformierung nicht daraus, dass die Zigeuner nie die gesellschaftlichen Regeln befolgen, sondern dass sie es meistens nicht tun: Es gibt Figuren, welche die Regeln des ehrenhaften Rittertums der Heldenliteratur befolgen, aber die Mehrheit der Zigeuner befolgt sie nicht. Es gibt unter ihnen einige Hochgelehrte, die sich auf raffinierte Weise über politische Philosophie ergehen, aber die meisten reden nur dummes Zeug: Manche bleiben dem Prinzen immer treu, aber die Mehrheit ist bereit, sich an die Türken zu verkaufen. Diese Charaktereigenschaft, die auch Lebarbier feststellt (1991, 184), führt dazu, dass wir bei den Zigeunern von Budai-Deleanu wieder die grundlegende Trickster-Eigenschaft finden: „alles in den Augen aller" zu wissen.

\section{DieZigeuner in der transnationalen Ethnologiedes20.J ahrhunderts}

Der Literaturspezialist Franchot Ballinger (1991, 2006) insistiert auf den bestehenden Unterschieden zwischen dem amerikanischen Trickster und dem euro-amerikanischen Picaro, auch wenn er die vielen gemeinsamen Eigenschaften nicht abstreitet. So ziehe Ersterer die Grenzen des Menschlichen in Zweifel, während Letzterer lediglich die herrschenden sozialen Grenzen zur Diskussion stelle. Ballinger tut recht, darauf hinzuweisen, dass es sich um Figuren aus sehr 
unterschiedlichen Universen handele. Aber genau aus diesem Grund ist die Unterscheidung nicht so wichtig: Es ist klar, dass in Gesellschaften, die keinen Unterschied zwischen Natur und Kultur machen, die Grenzen des Menschlichen selbst in Frage gestellt werden, während in Gesellschaften, für die dieser Unterschied konstitutiv ist, vor allem die sozialen Regelsysteme revisionsbedürftig erscheinen. Dadurch wird klar, warum die transkulturelle Kategorie des Trickster, die von den Ethnologen konstruiert wird, ziemlich mehrdeutig ist, man könnte sagen „tricksterhaft“, wie übrigens die meisten ethnologischen Kategorien (Piasere 2002). So könnte im folgenden Zitat Ballingers die Figur des Trickster sehr gut durch die Figur des Zigeuners, wie wir ihn bisher geschildert haben, ersetzt werden: „Like a subatomic particle, Trickster never allows final defi of time, place and character. He never settles or shapes himself so as to allow closure, either fictional or moral. We may believe that we have somehow fixed him at one moment, but if we look from another angle, he's gone; if we ask a different question, we get a different answer which, we must confess, is conterminus with the first. [...] He eludes all attempts to place him within the categories of definition and classifi [...] Similarly, Trickster's power to transform himself reinforces our perceptions of him as an image of manysidedness." (Ballinger 1991, 31-32)

Wenn man darüber nachdenkt, dass die komödiantischen Heldengedichte und die Zingaresche des Rinascimento, die „Ţiganiada“, und die spaßhaften rumänischen Erzählungen von der Menschheit und ihrer lokalen Klassifizierung berichten, so wird klar, dass sie vom Gegenstand der Anthropologie handeln, seit sich diese als wissenschaftliche Disziplin konstituiert hat. Wenn dem so ist, finden wir dann Spuren des Trickster in der anthropologischen Literatur selbst? Die Tsiganologie (oder Romalogie oder Romanologie) wurde als Forschungsfeld mehr oder weniger in den gleichen J ahrzehnten begründet wie die moderne Ethnologie, nämlich in der zweiten Hälfte des 19. J ahrhunderts. Dennoch werden die Roma und Sinti erst spät zum Gesprächsthema der internationalen Sozialwissenschaften. Es gab einige isolierte Vorreiter in den vorherigen J ahrzehnten, aber man kann sagen, dass sie erst in den 1970erJ ahren wirklich zum Thema werden. Und zwar mit einer Anthologie, die Farnham Rehfisch herausgab (1975) und Monographien über die nord-amerikanischen Rom kalderaš (Gropper 1975; Sutherland 1975; Salo/Salo 1977). Die wichtigsten Entwicklungen, deren Einfluss bis heute anhält, fanden in den 1980er J ahren statt. In dieser Zeit werden die Zigeuner mit den Trickster-Eigenschaften ausgestattet, natürlich in den Grenzen, die sich aus 
der neuen Art des Wissens und der Wissenskonstrukteure, die von ihnen sprechen, ergeben. Ich möchte auf zwei Punkte eingehen: der Platz der Zigeuner in der Welt, wie er von den Tsiganologen konstruiert wird, und einige Alltagspraktiken der Zigeuner, wie sie von den Forschern gesehen werden.

1983 schlägt J arold Ramsey vor, den indianischen Trickster-Verwandler weniger als kulturelle Heldenfigur zu betrachten, denn als „Bastler" im Sinne der berühmten Interpretation von LéviStrauss (1962, 26-33): Der Trickster sei „a sort of mythic handy-man who 'cobbles' reality in the form of bricolage out of the available material, and with something distinctly less transcendent than a divine plan or teleology to guide him, namely, his own impressionable, wayward, avid mind“ (Ramsey 1983, 41). Ebenfalls 1983 sagt J udith Okely, eine der Begründerinnen der zeitgenössischen sozio-kulturellen Zigeuner-Ethnologie, praktisch das Gleiche über die Zigeuner: „The Gypsies may indeed incorporate symbols, rites and myths from the larger society, but there is a systematic, not random, selection and rejection. Some aspects may be transformed or given an inverted meaning. The Gypsies [...] can be seen as bricoleurs, picking up some things, rejecting others. The ideology of the dominant society is de-totalised, and the ultimate re-synthesised cosmology takes on a new coherence with perhaps an opposing meaning, and one which accomodates the Gypsies as an independent group“ (Okely 1983, 77). Die englische Tsiganologin argumentiert in ihrer Erörterung gegen dieSichtweise einer in sich geschlossenen Zigeuner-Kultur einerseits, dass die Zigeuner sich der Aneignung kultureller Züge von außen nicht verschließen können und dass sie andererseits unter diesem Kontakt nicht als Minderheit leiden, sondern die Hoheit darüber behalten, und das trägt zur Wahrung ihrer Identität bei. Diese „Bastelei“ mit externen Elementen und interpretiert im Sinne von Mary Douglas’ Reinheitskonzept würde dazu dienen, die symbolischen Grenzen zur Gesellschaft im weiteren Sinne zu bewahren. Im selben Zeitraum sagt Patrick Williams im Grunde das Gleiche, ebenfalls mit der Metapher von LéviStrauss. In einem Artikel von 1981 hatte er bereits klar das Thema formuliert, das er in seiner Monographie von 1984 über die Roma in Paris wieder aufnahmen: „Konstitution und Verfall, das scheint mir das Motiv zu sein, das sowohl die soziale Organisation als auch die Kultur der Zigeuner auszeichnet. [...] Alle Zigeuner-Kulturen bestehen aus fremden Elementen (die ursprünglichen Züge sind indisch, nicht zigeunerisch) und sind geborgt) [...]. Erwerb und Aufgabe sind von der gleichen Ambivalenz gekennzeichnet, auch sie tragen zur Konstitution und zum Verfall bei. Das 
Eigene der Zigeuner-Kultur liegt in dieser Bewegung, in dieser Kombination, in diesem zerbrechlichen Gleichgewicht“ [7] (Williams 1981, 40). Der französische Tsiganologe unterstreicht im Rahmen der Diskussion um die „Zigeuner-Spezifi dass jede Zigeuner-Gruppe „mit geliehenen Elementen eine eigene Erscheinung“ [8] schaffen kann (ebd., 32). Er führt als Beispiele Sprache, Konzepte von Unreinheit und Musik an. Ich bin zu dieser Zeit selbst so vorgegangen und habe versucht zu zeigen, dass Zigeuner-Gruppen ihre Kultur mit importierten Elementen konstruierten: Eine Gruppe muslimischer Roma aus dem Kosovo beispielsweise konstruierte ihr Verwandtschaftssystem auf einer Blutsverwandtschafts-Terminologie „serbischen“ Typs, das Zusammengehörigkeitsgefühl „griechischen“ Typs und die Regeln der Vendetta „albanischen“ Typs (Piasere 1982, 1983, 1984). Einige J ahre später verwendete J ane Dick Zatta (1991) einen Ausdruck, der eher an den Trickster erinnert und sprach in Bezug auf die linguistische Struktur einer Gruppe slowenischer Roma von čordi čhip, „gestohlener Sprache“. Diese Studien waren mit Absicht anti-essentialistisch und standen im Zeichen des damaligen Überdenkens der Frage nach der Akkulturation der Zigeuner. Es war die Epoche, als der Neo-Diffusionismus der postmodernen Ethnologie noch nicht oder gerade erst geboren war. Es kommt nicht von ungefähr, dass die Roma-AutorInnen und -ForscherInnen (und Nicht-Roma), die nach grundsätzlichen Sicherheiten der „wahren Kultur indischen Ursprungs“ suchen, diese Vorgehensweise und ihre Ergebnisse nicht gut verdaut haben. Heute sprechen alle von „kulturellem Hybridismus“ (ein schrecklicher Ausdruck), damals waren die Zigeuner-AnthropologInnen und -EthnologInnen ziemlich isoliert mit ihrer hybriden „Kreatur“. Die „Zigeuner-Kultur“, die so zwischen den als „pur“ präsentieren Kulturen neu formuliert wurde, war doppelt tricksterhaft. Zuallererst besitzt sie die gleiche Un-Vorstellbarkeit, welche die Mythen dem Trickster zuschreiben, in ihrer permanenten Bereitschaft, die Form zu wechseln, womit sie „ungreifbar“ wird. Man sagte, die Zigeuner-Kultur sei „fl „geometrisch variabel“, oder ähnlich einem „Mosaik“ oder einem „Kaleidoskop“ mit immer wechselnden Formen-Metaphern, die sich später weit verbreitet haben und in der postmodernen Anthropologie und Ethnologie allgemein verwendet wurden. Zweitens gewann diese unvorstellbare Kultur ihre Gestalt durch geliehene „Stücke“, die aus verschiedenen Kulturen kamen und zusammengestellt oder neu formuliert wurden, bis neue Identitäten verschiedener Zigeuner-Gruppen konstruiert werden konnten. Allerdings wurde diese rekonstruierte ZigeunerKultur für die Analytiker so etwas wie das Schnabeltier, von dem Umberto Eco spricht (1997): Ein
[7] „Constitution-désintégration, telle semble bien être la figure qui caractérise tant l'organisation sociale que la culture tsiganes. [...] Toutes les cultures tsiganes ne sont faites que d'éléments étrangers (les traits d'origine sont indiens, pas tsiganes) et empruntés [...]. Acquis et abandon sont marqués de la même ambivalence, ils participent également de la constitution et de la désintégration. Le propre de l'identité tsigane est dans ce mouvement, cette combinaison, cet équilibre précaire."

[8] „phénomène original avec des éléments d'emprunt“ 
Tier, dass bei seiner Entdeckung im 18. J ahrhundert weder als Fisch noch Vogel noch Vierfüßler kategorisiert wurde, obwohl es morphologische Eigenschaften vom Fisch ebenso wie vom Vogel ebenso wie vom Vierfüßler hatte (mehr oder weniger wie unser Margutte). Blumenbach, einer der Väter der physischen Anthropologie, nannte es sogar Ornythorinchus paradoxus - ein Versuch, durch den Begriff „paradox“ „etwas zu kategorisieren, das unkategorisierbar war“ [9], bemerkt Eco (1997, 210). In der gleichen Weise, wie man schließlich übereinkam, die neue Ordnung der Kloakentiere zu schaffen, um das Schnabeltier zu klassifizieren, genauso wurden in den 1980ern verschiedene Vorschläge für die Schaffung einer neuen anthropologischen Kategorie gemacht, die auch die Zigeuner einschließen sollte. Am berühmtesten ist vermutlich die der Peripatic People, die Berland/Salo (1986) und Aparna Rao (1987) auf der Grundlage ökonomischer und ökologischer Überlegungen vorgeschlagen haben. Der Vorschlag wurde nicht von allen angenommen (vgl. Williams 1990). In einem internationalen Workshop in Paris 1991 unter dem Titel „Etudes des Tsiganes et questions d'anthropologie“ blieb das Thema des schwierigen Verhältnisses zum tsiganologischen Wissensvorrat zentral. In meinem Beitrag fragte ich mich, ob man die Zigeuner durch ihre taxonomische Anomalie in der Geschichte der westlichen Gedankenwelt „anthropologisch denken könne“ (Piasere 1994). J udith Okely (1994) betonte die Herausforderung, welche die Untersuchung der Zigeuner für die herrschende Hegemonie der akademischen Ethnologie oder Anthropologie darstelle: Die Zigeuner-Spezialisten seien akademische Außenseiter und könnten deshalb sehr innovative Vorschläge machen. Patrick Williams unterstrich, dass „wenn der Beitrag dieser Ethnologie der Zigeuner zur allgemeinen Ethnologie darin besteht, bestimmte Konzepte zu destabilisieren, dann liegt das daran, dass die Autoren immer angehalten waren, den Schwerpunkt auf die unfertige Seite der von ihnen analysierten kulturellen und sozialen Kreationen zu legen“ [10] (Williams 1994, 5). Ist nicht genau das die Rolle des Trickster? Das herrschende Weltbild zu destabilisieren, um zu zeigen, dass mit demselben kulturellen Material andere Weltbilder möglich sind, und um die taxonomischen und politischen Hegemonien kontinuierlich in Frage zu stellen?

Wie dem auch sei, die „Destabilisierung“ wird noch deutlicher, wenn es sich um konkrete Objekte handelt und nicht mehr um ethnologisch-anthropologische Konzepte. Miriam Kaprow, die in den 1970erJ ahren zwei J ahre bei einerZigeuner-Familiein Saragossa lebte, warverzweifelt, denn „unlike my role models of ethnographic fame, I could not locate anything among the Gypsies I had come
[9] „de catégoriser quelque chose en tant qu'incatégorisable“

[10] „Si l'apport de cette ethnologie des Tsiganes à l'ethnologie en général réside dans une certaine 'fragilisation' de tel ou tel concept, c'est parce que les auteurs ont été conduits à mettre l'accent sur le côté toujours inachevé des créations sociales et culturelles qu'ils analysaient." 
to study resembling a genuine group“ (Kaprow 1991, 218). Sie spricht von Personen, die in gleicher Weise „Permanenz und Struktur vermeiden“ (ebd., 225) und auch eine Tendenz zeigen, ihre materiellen Güter wie Kleidung, Möbel etc. nicht zu erhalten. So beschreibt Kaprow zum Beispiel, wie Kinder neue Sofas bekritzeln und ins Polster schneiden, ohne dass die Erwachsenen einschreiten. Die Destabilisierung, die ganz einfach eine Zerstörung oder Eliminierung materieller Güter sein kann, ist oft das offensichtlichste Phänomen, das zum Urteil eines nicht-rationalen Verhaltens der Zigeuner führt. Dies ist begründet in einem Gegensatz zum gesellschaftlichen Wertekonsens der Bewahrung von Gütern. Aus der Sicht der Nicht-Zigeuner verhält sich ein Zigeuner, der seine Wertgegenstände zerstört, genauso wie der Trickster der Winnebago, der seine eigenen Eingeweide isst. Am meisten beeindruckt dieses Phänomen die Soziologen (mehr als die Ethnologen): Ladányi und Szelényi (2005), zwei ungarische Soziologen untersuchten ein Roma-Dorf in Ungarn in der Übergangszeit zwischen Sozialismus und Kapitalismus. Auch sie haben das Problem der Klassifikation, und weil sie Myrdals Konzept der Underclass wieder haben auferstehen lassen, versuchen sie zu zeigen, dass diese Roma dafür ein frappierendes Beispiel sind. In diesem Sinne erzählen sie von Entwicklungshilfeprojekten, die völlig erfolglos im Dorf durchgeführt wurden. Die Autoren selber sprechen von „tragisch-komischen Szenen“; ich würde eher von Szenen der Ţiganiada sprechen:

- Roma werden als Holzarbeiter in einem Unternehmen angestellt. Weil sie aber davon ausgehen, ausgebeutet zu werden, lehnen sie es ab, zu arbeiten und verkaufen die vom Unternehmen bereit gestellten Äxte auf dem Schwarzmarkt. Ergebnis: Das Unternehmen beendet dieseArbeiten.

- Eine holländische Stiftung organisiert zu Gunsten der Roma eine Kleiderverteilung. Die Zigeunerjedoch stürzen sich auf die LKWs, sobald sieim Dorf eingefahren sind, und versuchen, die größstmögliche Menge Kleidung an sich zu bringen. Dann verlangen sie, die Verteilung müsse gerecht erfolgen und jeder müsse das gleiche Kleidungsstück, wenn möglich in der gleichen Farbe erhalten. Ergebnis: Die Kleiderverteilung wird eingestellt.

- Ein junger Holländer kommt ins Dorf mit der Absicht, dort eine Modell-Farm aufzubauen, und stellt Roma ein. Diese bestehen darauf, dass er Mitglieder jeder familiären Gruppe des Dorfes einstellt. Das Ergebnis: Die Produktion ist nur mittelmäßig und nicht rentabel. 
- Eine Kooperative pfl Kartoffeln an. Um zu vermeiden, dass die Roma die Felder plündern, sobald die Kartoffeln reif sind, lässt der Bürgermeister die Felder mit Wasser besprenkeln, gibt dabei aber den Roma zu wissen, es handele sich um ein gefährliches Pestizid, das einen monströsen Durchfall verursache, wenn es mit den Kartoffeln eingenommen werde. Ergebnis: Da die Roma die Täuschung nicht durchschauen, lassen sie die Kartoffeln unberührt.

- Als ein Dorf von einer schweren Krise erfasst wurde, verließen dieNicht-Zigeuner ihreHäuser, verkauften aber vorher Rohre und Türrahmen. Die Roma haben „diese Praktik ,perfektioniert': während die Bauern ihre Häuser zerstörten, wenn sie sie verließen, zerstören die Zigeuner ihre Häuser, während sie noch darin wohnen“ [11] (Ladányi/ Szelényi 2005, 85). Wenn es sehr kalt ist, verbrennen sie sogar ihre Fenster und Türen, um sich aufzuwärmen. Ergebnis: Am nächsten Tag frieren sie noch mehr.

Die Soziologen etikettieren die Handlungsweise mit dem Ausdruck „kurzfristige Rationalität", es könnte sich aber auch durchaus um eine Episode aus einer Trickster-Geschichte handeln...

\section{Horror infiniti}

Wir könnten sagen, dass die Trickster aus der Folklore die analogen Entsprechungen zu den irrationalen Zahlen in der Mathematik sind, also Zahlen, die nicht wie eine endliche Zahlenfolge geschrieben werden können, und keiner Ratio folgen: bis in die Unendlichkeit. Auch der Trickster kann nie mit einer endlichen Serievon Handlungen beschrieben werden: Erkann alles machen, ohne Ratio: bis in die Unendlichkeit. Und ist der Trickster nicht genau wie die Zahlen eine Erfindung des menschlichen Gehirns? Im Verhältnis zu den ungefähr $10^{80}$ existierenden Atomen kann das menschliche Gehirn ungefähr 10 $0^{70.000 .000 .000 .000}$ Gedanken fassen (Barrow 2005, 25): Darunter ist sicher auch ein Platz für die irrationalen Zahlen. Und für die Trickster...

Was uns hier interessiert, ist eine andere Analogie. Wenn es stimmt, dass die Mathematik im heutigen Okzident die Wissenschaft ist, die sich mit dem Studium der Unendlichkeit befasst, dann heißt das nicht automatisch, dass sie immer gut aufgenommen wurde. Die Unendlichkeit
[11] „Perfectionné cette pratique: si les paysans démantelaient leurs habitations en les quittant, les Gitans les détruisent tout en continuant à y vivre." 
hat ihre Toten gehabt: Man kennt die Geschichte, von der es heißt, als man die irrationalen Zahlen entdeckte und erkannte, dass sie alles auf den Kopf stellen würden, haben die Pythagoreer ihre Verbreitung verboten. Der Verräter, ein gewisser Hippase von Métaponte, wurde von Zeus verurteilt, bei einem Schiffbruch zu sterben. Man kennt die Leidenserfahrungen von Georg Cantor, der im Krankenhaus an einer Depression starb, die durch die Anfeindungen seiner Kollegen ausgelöst wurde. Erwähnen wir auch Giordano Bruno, der von der Existenz unendlicher Welten überzeugt war und dafür von der katholischen Inquisition verurteilt wurde, weil sie nicht eingestehen konnte, es könne etwas Unendliches außerhalb von Gott geben (den Gott, wie ich erinnern möchte, den Nicolaus Cusanus als „Vereinigung der Gegensätze“ beschrieben hatte: wie die Unendlichkeit, wie der Trickster...). Im westlichen Denken, so J ohn Barrow $(2005,3)$ besteht diese Angst vor der Unendlichkeit auch außerhalb der Mathematik und der Theologie: Er zitiert die Physik, für welche die Unendlichkeit ein Unglück ist. So bald ein Physiker in seinen Berechnungen auf etwas Unendliches stößt, ist er sicher, sich geirrt zu haben und tut alles, um einen anderen Weg zu finden.

Aber dieser horror infiniti existiert nicht nur in der Physik, sondern auch in der Gesellschaft und im täglichen Leben.

Welchen Unterschied gibt es zwischen den Trickstern und den Zigeuner-Trickstern? Kehren wir nach Rumänien zurück, wo, wie wir gesehen haben, der Versuch des Amalgams am explizitesten war. Welchen Unterschied gibt es zwischen Păcală und dem Zigeuner? Dass Păcală nur in der Mythologie existiert, während der Zigeuner in der Mythologie und im realen Leben existiert. Wie Micheline Lebarbier sagt, „der eine ist eine imaginierte Kreation, der andere hat seine Wurzeln in der Realität“ [12] $(1989,145)$. Auch wenn im Leben der Menschen der Unterschied zwischen dem Realen und dem Imaginären sehr nuanciert ist, ist etwas Wahres in den Worten der französischen Anthropologin. Das Unendliche denken ist eine Sache, ließe sich sagen, es zu praktizieren, eine andere. „In den Erzählungen von Păcală“, sagt uns noch einmal Lebarbier, „identifizieren wir uns mit Păcală, er sieht aus wie ein Depp, aber er hat in 1000 Situationen Erfolg, schafft Wirrwarr und löst ihn auf; im Falle des Zigeuners identifiziert sich der Zuhörer nicht mit dem Zigeuner, im Gegenteil, er fühlt sich in seinem eigenen Bild bestätigt und ist froh, kein Zigeuner zu sein. “ [13] (ebd., 145). Nach der Interpretation von Mihai Rezvan hat der „karikaturale, lächerliche und groteske“ Zigeuner die Funktion, die Normalität der Welt durch Umkehrung zu sanktio-
[12] „L'un est une création imaginaire, l'autre a ses racine dans le réel."

[13] „Dans les contes de Păcală - nous dit encore Lebarbier - on s'identifie à Păcală, sot en apparence mais dominant et réussissant dans mille situations, créant et dénouant des imbroglios ; dans le cas du Tsigane, l'auditeur ne s'identifie pas au Tsigane au contraire, il se trouve conforté dans sa propre image, satisfait de n'être pas Tsigane." 
nieren, denn er stellt eine Figur dar, die sich zu weit vom Ideal der akzeptierten Normen entfernt hat: „Durch die Repräsentation des Zigeuners, einer karikaturalen und entstellten Menschheit, haben die Bauern die Möglichkeit, ihr Konzept einer ,normalisierten’ Menschheit auszudrücken und rituell wie didaktisch ihr soziales und menschliches Ideal zu bestätigen. " [14] (Rezvan 1985, 116-117). Der rumänische Bauer wird einem Zigeuner tatsächlich begegnen, Păcală nicht. Und wie es die Narrative gewisser Ethnologen postulieren, konstruieren zahlreiche Zigeuner ihr Bild nach dem Bild, dass man sich von Trickstern macht: Bastler, kulturell hybrid, im Kampf mit den herrschenden Regelsystemen, Erkunder der Nicht-Ordnung, à la Savard wellenförmige Erschaffer ihrer eigenen Diversität ausgehend vom Kontinuum, das von den Nicht-ZigeunerKulturen repräsentiert wird, wie es Patrick Williams (1993) für die französischen Mānuš gezeigt hat, also Entweiher der Nicht-Zigeuner-Ordnung, Demo-Aristo-Monarchen, alles und nichts... Wie die Soziologen bestätigen, gibt es tatsächlich Zigeuner, welche die Art von Gedanken, die wir „Rationalität“ nennen, auf kurzfristige Weise anwenden, die nach unserer herrschenden Ideologie langfristig angewendet werden müsse. Was passiert, wenn der Trickster-Zigeuner außerhalb des Bereichs des Mythischen auftaucht, da, wo die mögliche Unendlichkeit sich als aktuelle Unendlichkeit darstellt? „Das menschliche Denken“ sagt J ohn Barrow „hat es geschafft, den Begriff der Unendlichkeit zu erfassen, um sich dann im Angesicht seiner Auswirkungen zurückzuziehen.“ [15] (Barrow 2005, 9) Im Fall der Zigeuner verwirklicht sich der Übergang vom Lachen zum Schrecken. Die Geschichte der Zigeunerverfolgungen in Europa - vergangene und aktuelle, seit der Zeit von Cingar über Auschwitz, wie es Bernhard Streck (1981) vor 30 J ahren dokumentiert hat, bis heute im Italien von Berlusconi - zeigt, was durch die Angst passieren kann, die vor der Materialisierung des Trickster herrscht, der mit seiner Ironie den Herrschenden und ihren Weltbildern die Leviten liest, und dabei immer bereit ist, seine Nicht-Subalternität zu bewahren, selbst wenn er verfolgt wird.

Aber wer sagt uns, dass dieser horror infiniti, dieser Schrecken vor dem Unendlichen, nicht der $\mathrm{x}$-te Streich des Trickster ist, seine x-te Aktion bei der Erkundung der Nicht-Ordnung?

(Übersetzung aus dem Französischen von Kathleen Schlütter)
[14] „Ainsi, l'humanité conçue par la représentation du Tsigane, humanité déformée et caricaturale, permet aux paysans d'exprimer leur conception d'une humanité 'normalisée' et d'affirmer rituellement et didactiquement leur idéal social et humain."

[15] „La pensée humaine est parvenue à embrasser la notion d'infini pour ensuite se retirer face à ses implications" 


\section{Bibliographie}

\section{LiterarischeQuellen}

Boiardo, M. M. (1978) Orlando Innamorato. A cura di G. Anceschi. Milano: Garzanti..

Budai-Deleanu, I. (1999) Ţiganiada. Ediţie critică de F. Fugariu, Timişoara: Amarcord.

Folengo, T. (1977) Opere. A cura di C. Cordiè. Milano \&Napoli: Ricciardi.

Pulci, L. (1955) Il Morgante. A cura di F. Ageno. Milano \&Napoli: Ricciardi.

\section{Sekundärliteratur}

Agamben, G. (1996) Mezzi senza fini. Torino: Boringieri.

Ballinger, F. (1991) Ambigere. The Euro-American Picaro and The Native American Trickster. In: Melus, 17(1): 21- 38.

Ballinger, F. (2006) Living Sideways: Tricksters in American Indian Oral Traditions. Norman: University of Oklahoma Press.

Barrow, J . D. (2005) L'infinito. Milano: Mondadori. (Ed. Or. 2005).

Berland, J . C./ Salo, M. T. (eds.) (1986) Peripatetic Communities. In: Nomadic Peoples, 21/22 (special edition).

Boas, F. (1898) Introduction. In: Teit, J . Traditions of the Thompson River Indians. Memoirs of the American Folklore Society, Vol. 6, 1-18. New York: Houghton Mifflin.

Brinton, G. D. (1868) The Myths of the New World. Philadelphia: Mckay Co.

Campigotto, A./ Piasere, L. (1990) From Margutte to Cingar: The Archeology of an Image. In: Salo, M. T. (ed.) 100 Years of Gypsy Studies. Cheverly (MD): Gypsy Lore Society, 15- 29.

Colli, G. (1998) Zenonedi Elea. Lezioni 1964- 1965. Milano: Adelphi.

Dick, Zatta (1991) Čordi čhip. Come i Roma hanno rubato la propria lingua. In: La ricerca folklorica, 22: 47- 54.

Eco, U. (1997) Kant el'ornitorinco. Milano: Bompiani.

Eliade, M. (1969) The Quest. History and Meaning in Religion. Chicago: University of Chicago Press. Evans-Pritchard, E. E. (1967) The Zande Trickster. Oxford: Clarendon Press.

Gombrich, E. H. (1986) L'eredità di Apelle. Studi sull'arte del Rinascimento. Torino: Einaudi, (Ed. or. 1966). 
Greco, F. (1976) Tradizione di una forma drammatica popolare: la 'Zingaresca' (con un documento inedito). In: Critica letteraria, 12: 516- 538.

Griaule, M./ Dieterlen, G. (1965) Le Renard pâle. Paris: Institut d’Ethnologie.

Gropper, R. C. (1975) Gypsies in the City. Princeton (NJ): Darwin Press.

Hamill, A. E. (1943) Scaramuccia, capitano de' Zingani. In: J ournal of the Gypsy Lore Society, 3rd series, 22(1/2): 56- 7.

Herskovits, M. J ./ Herskovits, F. S. (1958) Dahomean Narrative. Evanstone: Northwestern University Press.

J ung, C./ Kerényi, K./ Radin, P. (2006) Il briccone divino. Milano: SE. (Ed. or. 1954).

Kaprow, M. L. (1991) Celebrating Impermanence: Gypsies in a Spanish City. In: DeVita, P. R. (ed.) The Naked Anthropologist. Belmont (CA): Woodsworth, 218- 231.

Ladányi, J ./ Szeléni, I. (2005) La formation d'un sous-prolétariat rom. In: Actes de la recherche en sciences sociales, 160: 67- 88.

Kerényi, K. (2006a) Prefazione all'edizione italiana. In: Jung, C./ Kerényi, K./ Radin, P. Il briccone divino. Milano: SE (Ed. or. 1954), 9-21,

Kerényi, K. (2006b) Epilegomeni mitologici. In: J ung, C./ Kerényi, K./ Radin, P. Il briccone divino. Milano: SE (Ed. or. 1954), 135- 154.

Koepping, K.-P. (1985) Absurdity and Hidden Truth: Cunning Intelligence and Grotesque Body Images as Manifestations of the Trickster. In: History of Religions, 24(3): 191- 214.

Leach, E. (1980) L'unité del'homme. Paris: Gallimard.

Lebarbier, M. (1980) Păcală ou la fortune de l'absurde. In: Cahier de Litterature Orale, 7: 95- 116.

Lebarbier, M. (1989) L’identité dénaturée. Păcală et le Tsigane dans les contes facétieux roumains. In: De Sivers, F. (ed.) Questions d'identité. Paris: Peeters/ SELAF, 133- 150,

Lebarbier, M. (1991) Tsiganiada. Le Tsigane dans l'épopée de Ion Budai-Deleanu et dans les contes facétieux roumains. In: Cahier de Littérature Orale, 30: 177- 201.

Lévi-Strauss, C. (1958) Anthropologie structurale. Paris: Plon.

Lévi-Strauss, C. (1962) La pensée sauvage. Paris: Plon.

Lowie, R. H. (1909) The Hero-Trickster Discussion. In: J ournal of American Folk-Lore, 22: 431- 433. Maor, E. (1993) All'infi e oltre. Storia culturale del concetto di infi . Milano: Mursia (Ed. or. 1987). 
Meletinsky, E. M. (1973) Typological Analysis of the PaleoAsiatic Raven Myths. In: Acta Ethnographica Academiae Scientiarum Hungaricae, 22(1/2): 107- 155.

Miceli, S. (2000) Il demiurgo trasgressivo. Studio sul trickster. Palermo: Sellerio.

Mimica, J . (1992) Intimations of infinity. The cultural meaning of the Iqwaye counting and number system. Oxford: Berg. (Ed. or. 1988).

Münster, S. (1559) Cosmographia Universalis. Basileae: Heinrich Petri. (Ed. or. 1550).

Okely, J . (1983) The Traveller-Gypsies. Cambridge: Cambridge University Press.

Okely, J . (1994) L'études des Tsiganes: un défi aux hégémonies territoriales et institutionnelles en anthropologie. In: Études tsiganes, 2: 39- 58.

Partington, R. (1956) The Gypsy and the Holy Family. In: J ournal of the Gypsy Lore Society, 3rd series, 35(1/2): 1- 11.

Pettazzoni, R. (1953) Miti eleggende. Vol. 3, Torino: UTET.

Piasere, L. (1982) La terminologie des parents consanguins chez deux groupes rom. In: Études tsiganes, 2: 1- 24.

Piasere, L. (1983) I plešnóra. Uomini di pace fra i Xoraxané. In: Lacio drom, 1: 10- 26.

Piasere, L. (1984) Sistemi rom e sistemi gagé della terminologia dell'affinità. In: Lacio drom, 1: 6- 26.

Piasere, L. (1994) Les Tsiganes sont-ils "bonsà penser" anthropologiquement? In: Étudestsiganes, 2: 19- 38.

Piasere, L. (2002) L'etnografo imperfetto. Esperienza e cognizionein antropologia. Roma/ Bari: Laterza.

Piasere, L. (2005) La schiavitù dei rom in Moldavia. In: Solinas, P. G. (ed.) La dipendenza. Antropologia delle relazioni di dominio. Lecce: Argo, 287- 331.

Piasere, L. (2006) Buoni da ridere, gli zingari. Saggi di antropologia storico-letteraria. Roma: CISU.

Piasere, L. (2009) Il trickster el'infinito. Alcune riflessioni a partire da esempi rom. Communication présentée au colloque international “Pulcinella. L'eroe comico nell'area euromediterranea", Naples, 1- 3 juillet.

Proust, M. (1988) À l'ombre des jeunes filles en fleurs. Paris: Gallimard. (Ed. or. 1919).

Radin, P./ Kerényi, K./J ung, C. G. (1954) Der göttliche Schelm. Ein indianischer Mythen-Zyklus. Zürich: Rhein-Verlag.

Radin, P. (1953) The World of Primitive Man. New York: Schuman.

Radin, P. (1956) The Trickster. A Study in American Indian Mythology. Commentaries by Karl Kerényi and C. G. J ung. New York: Schocken Books. 
Radin, P. (2006) I Winnebago e il ciclo del briccone. In: J ung, C. G./ Kérenyi, K./Radin, P. Il briccone divino. Milano: SE (ed. or. 1954), 93- 133.

Ramsey, J . (1983) Reading the Fire. Essays in the Traditional Indian Literatures of the Far West. Lincoln: University of Nebraska Press.

Rao, A. (Ed.) (1987) The Other Nomads. Köln: Böhlau.

Rehfisch, F. (Ed.) (1975) Gypsies, Tinkers and Other Travellers. London: Academic Press.

Rezvan, M. (1985) Les paysans, les tsiganes et l'État-nation: le destin littéraire et politique d'un récit populaire roumain. In: C.N.R.S. (ed.) Paysans et nations d’Europe centrale et balkanique. Paris: Maisonneuve et Larose.

Róheim, G. (1950) Psychoanalysis and Anthropology. New York: International Universities Press.

Savard, R. (1978) Structures sémantiques et mythologie. Le personnage du décepteur dans la littérature orale amérindienne. In: Avalle, S. et al. Strutture e generi delleletterature etniche. Palermo: Flaccovio, 27- 38.

Senatore, A. (2006) Ion Budai-Deleanu. Interessi linguistici eimpegno letterario. Bari: Cacucci.

Sperber, D. (1975) Pourquoi les animaux parfaits, les hybrides et les monstres sont-ils bons à penser symboliquement? In: L'Homme, 15(2): 5- 34.

Salo, M. T./ Salo, S. (1977) The Kalderaš in Eastern Canada. Ottawa: National Museum of Canada. Streck, B. (1981) Zigeuner in Auschwitz. Chronik des Lagers B II e. In: Münzel, M./ Streck, B. (Hg.)

Kumpania und Kontrolle. Moderne Behinderungen zigeunerischen Lebens. Giessen: Focus, 69- 128. Sutherland, A. (1975) Gypsies. The Hidden Americans. London: Tavistock.

Tetel, M. (1963) Rabelais and Folengo. In: Comparative Literature, 15(4): 357- 364.

Williams, P. (1981) La société. In: Liégeois, J .-P. (ed.) Les populations tsiganes en France. Paris:

Centre de Recherches Tsiganes, 27-44.

Williams, P. (1984) Mariage tsigane. Paris: L'Harmattan \& SELAF.

Williams, P. (1990) Nomadisme et situation minoritaire: un essai de categorization. In: Études Rurales, 120: 171- 174.

Williams, P. (1993) Nous, on n'en parle pas. Paris: Éditions de la Maison des sciences del'homme. Williams, P. (1994) Introduction. In: Études tsiganes, 2: 4- 7.

Zellini, P. (1980) Breve storia dell'infinito. Milano: Adelphi.

Buletin, L., 2017. Berita Terkini. [Online] Available at: http://www.buletinlokal.com/ 